\title{
Regards croisés entre projections démographiques et gestion des questions de population au Niger
}

\section{ZOURKALEINI Younoussi}

\author{
Université de Zinder, Niger Republic \\ younoussi@gmx.com
}

\section{RESUME}

Après la mise en œuvre des politiques de population, depuis plus de vingt ans, le Niger détient le triple record en termes de plus forte fécondité, plus fort taux de croissance démographique et plus bas niveau d'indice du développement humain. Ainsi la maitrise de la croissance démographique préconisée n'est pas assurée et les hypothèses sur lesquelles se base les projections démographiques deviennent non probantes. Pour mettre en évidence cette contre-performance de ces politiques de population et préciser les orientations nouvelles, cette étude présente les écarts observés entre l'indice synthétique de fécondité projeté et celui observé, montre les enjeux sociaux économiques et politiques associés aux nouvelles projections démographiques du système des Nations Unies et suggère des champs d'actions possibles en termes de politiques et programmes à mettre en œuvre pour assurer une maitrise de la croissance de la population.

Mots clés : Projections démographiques, enjeux politiques, Niger.

Look met between demographic projections and management of the questions of population in Niger

\section{Abstract}

After the implementation of the politics of population, for more than twenty years, Niger detains the triple record in terms of higher fertility, higher demographic growth rate and lower level of index of the human development. So the control of the recommended population growth is not to assure and the hypotheses on which bases itself the demographic projections become not convincing. To highlight this disappointing performance of these politics of population and specify the new orientations, this study presents difference observed between the synthetic index of fertility planned and observed, shows the economic and political social stakes associated to the new demographic projections of the United Nations System and suggests possible radiuses of action in terms of politics and programs to implement to assure a control of the growth of the population.

Keywords: Demographic projections, political stakes, Niger.

\section{Introduction}

Les enjeux sociaux, économiques et politiques associés aux diverses trajectoires démographiques que l'on peut imaginer en analysant correctement les données disponibles dans un pays sahélien comme le Niger sont entre autres: sa capacité à satisfaire les besoins essentiels de sa population en santé et en éducation ; la possibilité ou non pour les systèmes agraires de réduire l'insécurité alimentaire et nutritionnelle en milieu urbain comme en milieu rural ; la capacité de sa population et des autorités à élaborer et à mettre en œuvre des politiques efficaces en vue d'un développement durable (Guengant, 20II).

De façon à éclairer précisément la situation démographique du Niger, une série de projections démographiques ont été réalisées après l'adoption de plusieurs horizons temporels. Les premières et deuxièmes projections démographiques par âge et sexe réalisées au Niger couvrent respectivement les périodes 1994-2025 et 2005-2050. Les deuxièmes actualisent les premières suite à la réalisation du troisième recensement général de la population et de l'habitat réalisée en 200 l et l'analyse des données des diverses nouvelles enquêtes réalisées entre 1994 et 2005 dont les résultats ont fait apparaitre la nécessité d'une actualisation des premières projections démographiques.

Aujourd'hui encore, avec la publication des résultats du quatrième recensement général de la population et de l'habitat, réalisé en 2012 , et ceux de nouvelles enquêtes réalisées au cours des dix dernières années les résultats appellent à l'actualisation des connaissances sur la population 
totale du Niger, ses principales caractéristiques et sur son dynamisme à l'avenir car les résultats des deuxièmes projections démographiques de la période 2005-2050 deviennent obsolètes au vue des résultats issus de ces nouvelles données disponibles.

En effet, de nouveaux résultats de projections démographiques sont indispensables pour le suiviévaluation et la révision des politiques et programmes sectoriels en cours d'exécution. II s'agit en particulier de l'atteinte des objectifs du développement durable, du programme de développement économique et social et des divers programmes qui l'accompagnent dans les domaines agricole, sanitaire, éducatif, etc...

Rappelons aussi que les projections démographiques sont définies comme un ensemble de scénarios imaginant l'effectif futur d'une population en fonction de certaines hypothèses faites sur la mortalité, la fécondité et les migrations. Au cours des deux premières projections démographiques ces hypothèses se sont appuyées fortement sur les résultats attendus à la suite de la mise en œuvre des politiques de population. En principe, si les objectifs des politiques de population sont atteints, pour une même période, des écarts réduits devraient être observés entre les résultats des projections et ceux qui sont révélés par des enquêtes postérieures. Des écarts importants, pour une même période, signifieraient que les objectifs des politiques de population mises en œuvre ne sont pas atteints.

Ainsi, notre premier objectif est de vérifier, pour une même période, si les résultats des projections démographiques s'éloignent de ceux des enquêtes et recensements, réalisés des années après leurs publications. Le deuxième objectif est d'analyser les récentes projections démographiques des Nations Unies qui constituent une référence dans le monde, y compris pour le Niger, car elles respectent une rigueur scientifique certaine. Le troisième et dernier objectif est de proposer des options politiques qui permettront de réduire à l'avenir les écarts entre les résultats projetés actuellement et ceux qui seront issus des opérations de collecte de demain.

La présente étude s'articule autour d'une brève aperçue des options politiques pour une réduction de l'accroissement général de la population ; une présentation de la méthodologie et des anciennes projections démographiques puis les principaux résultats et enjeux des nouvelles projections démographiques des Nations Unies et enfin le dernier point aborde les champs d'actions possibles en termes de politiques et programmes à mettre en œuvre.

Revue de la Littérature et Cadre Théorique

Aujourd'hui encore, la querelle est vive entre ceux qui clament les bienfaits de la croissance 2932 démographique et ceux qui ne rêvent que d'une réduction de la croissance démographique. Elle n'est pas nouvelle. Cependant l'idéologie dominante en la matière a évolué dans le temps.

On peut souligner que cette querelle qui a tant durée vient en partie de la coexistence de deux situations complètements différentes: celle des pays du tiers monde dont les programmes de développement se heurtent à une croissance démographique trop rapide, qui conforte les thèses malthusiennes et; celle des pays industriels menacés d'une dépopulation et dont la population vieillit rapidement, qui appelle les réactions natalistes (Vallin, 1995).

Cependant, point n'est besoin de théorie pour admettre que le maintien de taux de croissance démographique de $3 \%$ ou $4 \%$ dans les pays du tiers monde conduiraient à la catastrophe et souhaiter leur modération aussi rapide que possible; ni pour reconnaitre que le prolongement de la baisse de la fécondité conduirait in fine les pays industrialisés à leur disparition et souhaiter le retour au point d'équilibre (Vallin, 1995).

Pour ralentir la croissance démographique rapide, selon Potts et al. (20I2), deux grandes options un peu contradictoires existent (Tableau I). L'une met l'accent sur l'amélioration socioéconomique, dans laquelle beaucoup voit la clé du déclin de la fécondité. L'autre consiste à accorder une plus grande importance à la possibilité de permettre à chaque couple de pouvoir planifier ses naissances en tenant comptes de ses possibilités à pouvoir élever sa descendance dans de très bonnes conditions.

Les partisans de l'option de l'amélioration socioéconomique continuent de penser ou de croire que le développement est le facteur principal de la transition de la fécondité et n'accordent souvent qu'un appui symbolique à l'investissement dans la planification familiale. Ceux qui soutiennent cette option de l'amélioration socioéconomique se subdivise en deux groupes : le premier qui opte pour une politique dite de "solution du marché» et le second qui opte pour une politique dite «investissement limité».

Pour les partisans de la solution du marché, il faut principalement voir dans une fécondité élevée le reflet du nombre d'enfants désiré reposant sur les avantages et désavantages pour un couple d'avoir un enfant. En effet, les avantages sont liés au plaisir que l'enfant procure aux parents, à son apport économique, à la garantie de la sécurité en cas de vieillesse des parents. Par contre, le coût élevé de son éducation et les coûts d'opportunités que la mère perd en accouchant, font que les parents renoncent le plus souvent à une descendance nombreuses. Ce qui emmène le plus souvent les http://aps.journals.ac.za 
parents à prendre une décision rationnelle en tenant compte des coûts et opportunités de la naissance d'un enfant. Mais cela suppose que les options contraceptives soient généralement accessibles et que la population soit suffisamment informée pour choisir en connaissance de cause.

La politique de l'investissement limité accorde la plus grande importance aux facteurs socioéconomiques de la transition vers les familles moins nombreuses, mais peut aussi parfois inclure un léger investissement dans la planification familiale à titre d'équité. Cette politique exclut explicitement toute considération de ralentissement de la croissance démographique. Ainsi les services de planification familiale doivent être assurés dans le contexte de programmes de santé reproductive globaux dont l'objectif fondamental est l'amélioration de la santé, et non la réduction de la fécondité ( Hodgson et Watkins, 1997).
La politique de recours à la planification familiale est celle mettant l'accent sur la planification familiale mais qui ne nie nullement l'importance de l'investissement dans le capital humain, l'amélioration de la santé, la réduction de la pauvreté et l'amélioration de la condition féminine. Elle affirme plutôt que ces investissements seuls ne réduiront pas la croissance démographique rapide dans un avenir proche. Cette politique pose aussi qu'une attention stratégique vigoureuse à la planification des naissances est nécessaire, bien que non suffisante, au développement socioéconomique. Améliorer l'accès à une grande variété de méthodes de planification familiale permet de réduire le nombre de grossesses non désirées, la mortalité maternelle et infantile et ralentit la croissance du nombre de jeunes personnes à charge.

Tableau I : Options Politiques pour une gestion des questions de population.

\begin{tabular}{|c|c|}
\hline Options & Raisonnements \\
\hline \multicolumn{2}{|l|}{ Amélioration socio-économique } \\
\hline Solution du marché & $\begin{array}{l}\text { Le taux de fécondité reflète le choix des couples et aucun } \\
\text { changement de politique n'est nécessaire }\end{array}$ \\
\hline Investissement limité & $\begin{array}{l}\text { En mettant l'accent sur l'amélioration de la situation sanitaire et } \\
\text { des conditions socioéconomiques ont aboutira au déclin des taux de } \\
\text { fécondité }\end{array}$ \\
\hline \multicolumn{2}{|l|}{ Planification des naissances } \\
\hline Accès à la planification familiale & $\begin{array}{l}\text { Une programmation spéciale visant à améliorer l'accès à la } \\
\text { contraception fera baisser les taux de fécondité et est indispensable } \\
\text { au développement socioéconomique }\end{array}$ \\
\hline $\begin{array}{l}\text { Accès à la planification familiale et } \\
\text { augmentation de l'âge au premier } \\
\text { mariage }\end{array}$ & $\begin{array}{l}\text { Dans une société où la polygamie est admise et où l'âge au } \\
\text { premier mariage est précoce, des politiques de rehaussement de } \\
\text { l'âge au premier mariage doivent être complémentaire à l'accès à la } \\
\text { planification familiale. }\end{array}$ \\
\hline
\end{tabular}

Source : Malcolm Potts et al. (20/2)

Tout comme les politiques de l'amélioration socioéconomique, les politiques qui mettent l'accent sur la planification des naissances se subdivisent en deux catégories. La première cherche à répondre aux besoins non satisfaits de planification familiale et souligne que l'accès à la contraception et à l'avortement médicalisé peut assurer des familles moins nombreuses, même dans les communautés analphabètes et pauvres (Potts et al.2009). La seconde reconnaît que si de telles politiques peuvent probablement suffire à réduire le nombre d'enfants dans de nombreuses communautés, certains pays doivent également faire face au problème du mariage précoce dont il faut tenir compte (Bruce et Bongaarts, 2009).

Parlant du Niger, depuis 1992, ce pays a adopté et mis en œuvre une politique de population visant entre autres à maitriser sa croissance démographique et à augmenter la prévalence de la contraception. Aussi, avec l'adoption, lors de la Conférence internationale du Caire en 1994, d'une nouvelle approche globale centrée sur les droits reproductifs, ce pays a également adopté des politiques, plans, et programmes en matière de santé, de santé de la reproduction et de sécurisation des produits de santé de la reproduction. On peut noter que pour tenir compte de ces évolutions la première politique de population a été révisée en 2007.

Cependant, aujourd'hui encore, le Niger détient le double record mondial : forte fécondité: 7,6 enfants par femme. (INS et ICF International, 20I3) ; forte croissance démographique: $3,9 \%$ (selon les résultats du dernier recensement général de la population). 
Le Niger est aussi l'un des pays les plus pauvres au monde: $60 \%$ des ménages sont paurres selon les résultats de l'Enquête National Budget Consommation de 2007-2008 et il est classé dernier pays du monde sur la base de l'Indice du Développement Humain: $187^{\text {ème }}$ sur 187 depuis 2012 d'après les différents rapports du PNUD.

II convient donc de s'interroger sur l'impact qu'ont eu les politiques de population sur l'évolution démographique de ce pays. En d'autres termes, ces politiques, la première et la deuxième, jusqu'à quel point ont-elles contribué à la maitrise de la croissance démographique et à l'augmentation de la prévalence de la contraception dans ce pays ? Avant de tenter de répondre à cette question examinons l'approche méthodologique.

\section{Données et Méthodes}

Au Niger, la politique de population de 1992 affirmait que « la politique de population fait partie intégrante de la politique de développement économique et social " du pays, et que "son but essentiel est la maitrise de la croissance démographique et des flux migratoires, mais aussi d'assurer l'adéquation entre la croissance démographique et le développement économique ". Plus tard, en 2007, pour remplacer cette politique, le gouvernement adopte une " Déclaration du Gouvernement en matière de Politique de Population "(DGPP), dont l'objectif global est de " contribuer à la réduction de la pauvreté grâce à l'acquisition d'une mentalité et de comportements reproductifs aptes à induire au sein des populations une augmentation significative de l'utilisation de la contraception et une réduction des mariages précoces».

Si donc le Niger détient le triple record en termes de plus forte fécondité, plus fort taux de croissance démographique et plus bas niveau d'indice du développement humain, nous supposons que la mise en œuvre des politiques de population n'a pas pu permettre d'obtenir une maitrise de la croissance démographique ni une réduction de la fécondité et une augmentation du taux de prévalence contraceptive.

Pour vérifier cette hypothèse nous utilisons les résultats des projections démographiques que nous comparons avec ceux des opérations de collecte (Recensement Général de la Population, Enquête Démographique et de Santé....) réalisées des années après la publication des résultats projections démographiques. En effet, comme les hypothèses des projections démographiques s'appuient fortement sur les résultats attendus à la suite de la mise en œuvre des politiques de population, en principe si les des objectifs des politiques de population sont atteints, des écarts réduits devraient être observés entre ceux prévus et ceux observés pour une année donnée.

Par ailleurs, au regard des données actuelles disponibles nous tentons d'appréhender l'ampleur des efforts qui restent à accomplir, a travers une analyse des résultats des nouvelles projections démographiques qui devraient nous permettre de mettre en évidence à la fois les tendances lourdes, incontournables, auxquelles il faudra bien s'adapter et les marges de manœuvre possibles pour influencer la dynamique démographique et satisfaire plus ou moins bien les besoins des populations.

Les données utilisées pour réaliser cette étude comprennent les données publiées par l'institut National de la Statistique, la Direction Nationale de la Population et la Division de la Population des Nations Unies. II s'agit donc d'une comparaison, d'une analyse et d'une interprétation de données secondaires. En ce qui concerne les politiques, les plans et programmes dans les domaines de la population, de la santé, de la santé de la reproduction, ou de la réduction de la pauvreté et autres nous avons utilisé les documents originaux émanant des ministères en charge de la population, de la santé publique et de l'économie et des finances.

\section{Résultats}

Les premières projections démographiques (19942025) ont été réalisées par la Direction Nationale de la Population avec l'appui technique du Centre d'Etudes et de Recherches sur les Populations Africaines et Asiatiques(CERPAA). Elles étaient réalisées deux ans après l'adoption de la politique nationale de population de 1992. Ainsi, en espérant des effets significatifs de la mise en œuvre de la politique de population, il avait été retenu une baisse continue de la fécondité ; une augmentation annuelle de I,5 points du pourcentage de femmes en union utilisant une méthode de contraception ; une diminution de $90 \%$ en 1994 à $85 \%$ en 2024 du pourcentage des femmes en union d'âge compris entre 15 et 49 ans; une augmentation de l'espérance de vie d'environ I,5 ans tous les cinq ans (Niger, 1994).

Les résultats des diverses enquêtes réalisées au cours des années après ces projections, en particulier ceux de l'Enquête Démographique et de Santé du Niger (EDSN) de 1998, et ceux du troisième recensement général de la population et de l'habitat, réalisé en 200I, permettent de constater que la baisse rapide de la fécondité, imaginée en 1994, ne s'est pas produite. Le nombre moyen d'enfants par femme estimé à 7,4 enfants en 1992 s'est maintenu, contrariant ainsi la maitrise de la croissance démographique escomptée à partir de 1994 sous l'optimisme que les autorités politiques se sont 
engagés résolument dans la mise en œuvre de la politique de population adoptée en 1992.

Les raisons avancées pour expliquer cette contreperformance relèvent des contraintes financières et la grande instabilité politique des années 1990, (les coups d'Etat de 1996 et de 1999 ont entrainé l'arrêt de l'aide internationale pendant un certain temps) qui ont fortement perturbé la mise en œuvre de la politique de population et des programmes qui lui étaient associés.

Les deuxièmes projections démographiques (2005-2050) étaient réalisées par une équipe du Bureau Central du Recensement (BCR) avec l'appui technique et financier du Fonds des Nations Unies pour la Population (UNFPA). La démarche générale adoptée, fortement appréciable, comporte deux grandes options. La première considère la fécondité comme une variable dépendante d'un certain nombre de déterminants proches, en l'occurrence, l'utilisation de la contraception, moderne et traditionnelle, la proportion des femmes en union, la durée de l'insusceptibilité post-partum et la stérilité primaire (Niger, 2005).

La seconde option considère la fécondité comme une variable indépendante. Autrement dit, c'est l'objectif volontaire de réduction du nombre moyen d'enfants par femme qui détermine les efforts à fournir par rapport aux déterminants proches de la fécondité. Par exemple, l'on peut essayer de répondre à la question suivante : si l'on souhaite passer de 7 à 5 enfants par femme de 2005 à 20/5, quel niveau de prévalence contraceptive, faudrait-il atteindre pour réaliser cet objectif de fécondité ?

La démarche fait ainsi recours à trois types de scénarii d'évolution combinant diverses hypothèses notamment, le scénario tendanciel, le scénario de réduction rapide de la croissance démographique, le scénario dit " d'appel à l'action " (Niger, 2005). Pour le scénario tendanciel on a retenu une augmentation annuelle de 0,5 point de pourcentage de la prévalence de la contraception durant la période 2005 à 2050. Cela devrait conduire à une fécondité qui passerait de 7,2 enfants par femme en 2005 à 6,9 en 2015 puis à 5,8 en 2050 . Pour le scénario réduction de la croissance démographique, on s'est fixé des niveaux de fécondité jugés souhaitables pour réduire rapidement la croissance démographique : 5,4 et 3 enfants par femme respectivement, en 2015, 2025 et 2050. Pour le scénario " appel à l'action » on a retenu, pour l'ensemble de la période 2005 à 2050, une augmentation annuelle de la prévalence contraceptive de I point de pourcentage. Le nombre moyen d'enfants par femme passerait de 7,2 en 2005, 5,6 en 2015 et 2,7 en 2050 (Niger, 2005).
Les trois scénarii retenus ont en commun une hypothèse unique d'évolution de la mortalité associée à une hypothèse de maîtrise de l'épidémie du $\mathrm{VIH} /$ sida. On a supposé que l'espérance de vie à la naissance augmenterait de I,7 ans par période de cinq ans au cours de la période 2005-2050. Ce rythme de progression de l'espérance de vie intègre une hypothèse d'évolution de l'épidémie du $\mathrm{VIH} / \mathrm{sida}$ au Niger (une légère progression de la séroprévalence jusqu'à un maximum de $1,9 \%$ en $20 \mathrm{I}$, puis une diminution régulière jusqu'à atteindre $0,2 \%$ en 2050). S'agissant des migrations internationales, l'hypothèse unique concernant les trois scénarii est celle d'un solde migratoire nul (Niger, 2005).

Les résultats des différentes enquêtes réalisées depuis lors, en particulier ceux de l'EDSN 2006 et 2012 , et ceux du quatrième recensement général de la population et de l'habitat, réalisé en 20/2, indiquent que le scénario tendanciel n'est pas à l'ordre du jour encore moins celui d'appel à l'action ou encore celui d'une réduction rapide de la croissance démographique. La fécondité semble être en croissance $(7, I$ enfants en moyenne par femme en 2006 et à 7,6 en 2012) conduisant ainsi à un niveau de croissance démographique jamais égalé $(3,9 \%)$.

Dans ce contexte on peut souligner que l'objectif global assigné à la "Déclaration du Gouvernement en matière de Politique de Population ", de 2007, qui est de " contribuer à la réduction de la pauvreté grâce à l'acquisition d'une mentalité et de comportements reproductifs aptes à induire au sein des populations une augmentation significative de l'utilisation de la contraception et une réduction des mariages précoces » est très loin d'être atteint.

On comprend aisément que cette actualisation de la politique nationale de population n'a pas amener les autorités publiques, les autres acteurs nationaux, ainsi que les partenaires au développement à s'engager de manière claire et résolue dans les trois directions qui avaient été mises en exergues : I) un engagement fort en faveur d'un programme de santé de la reproduction et de planification familiale répondant aux besoins de la population ; 2) un engagement clair en faveur de la lutte contre les mariages précoces ; 3) une revalorisation de la pratique de l'allaitement maternel.

Les enjeux sociaux, économiques et politiques associés aux diverses trajectoires démographiques que l'on peut imaginer pour le Niger, en analysant les nouvelles projections démographiques des Nations Unies de 2014, sont énormes. En effet, il ressort du tableau 2, qu'indépendamment de l'hypothèse émise, la population du Niger dépassera 19 millions d'habitants en 2016. En 2020, les résultats indiquent que l'hypothèse de forte fécondité conduirait à des 
chiffres de population qui s'éloigneront de ceux obtenus à partir des autres hypothèses avec une différence de près d'un million d'habitants. Ainsi la population serait d'environ 24 millions dans le premier cas et d'environ 23 millions dans le second cas.

Avec une population de 17,3 millions d'habitants recensés au Niger en 20I2, on pourrait dire qu'indépendamment de l'hypothèse émise, un doublement de la population pourrait être observé au plus tard en 2030.

En 2040, sous l'hypothèse d'une faible fécondité la population du Niger atteindra 47 millions d'habitants contre 55 millions en l'absence de changement. Pour l'hypothèse de fécondité médiane et celle de fécondité forte la population serait de 50 et 53 millions respectivement. Avant que le recensement général de la population et de l'habitat ne révèle cette augmentation du taux de croissance général de la population $(3,3 \%$ à $3,9 \%)$ toutes les projections réalisées (Nations unies, projections nationales) laissaient croire que ce seuil ne pourrait être atteint qu'en 2050, soit dix ans plus tard.

La forte croissance démographique, observée au cours de la période $200|-20| 2$, pourra conduire à une population du Niger qui serait de plus de 63 millions en 2050 et cela indépendamment de l'hypothèse émise, soit une multiplication par quatre de son effectif actuel. On peut faire remarquer qu'avec une fécondité constante elle atteindrait 86 millions en 2050, soit une multiplication par cinq.

Comment le Niger va pouvoir nourrir une population qui va passer de 19 millions d'habitants aujourd'hui (2016) à plus de 63 millions en 2050, dans un contexte de terre semi-aride face à un changement climatique, ne nous semble pas clair. En effet, le Niger présente l'une des situations écologiques les plus fragiles au monde. Douze pour cent seulement de la surface du Niger bénéficie actuellement de pluies suffisantes pour soutenir l'agriculture. D'après les prédictions (Banque Mondiale, 2005), il n'en restera rien d'ici 2100. Le sol nigérien est pauvre en éléments nutritifs et il est soumis à une dégradation liée à l'érosion, au surpâturage et à une gestion souvent considérée comme non adéquate (Baidu-Foson et Napier, 1998). Depuis plusieurs décennies, le rendement agricole diminue d'une moyenne de $0,6 \%$ par an (Banque Mondiale, 2005).

Tableau 2 : Evolution de la population totale(en million) selon l'hypothèse émise.

\begin{tabular}{|l|c|c|c|c|c|c|c|c|}
\hline Population totale & \multicolumn{1}{|c|}{2015} & \multicolumn{1}{c}{2020} & 2025 & 2030 & 2035 & 2040 & 2045 & 2050 \\
\hline Fécondité médiane & 19268 & 23422 & 28477 & 34513 & 41589 & 49756 & 59038 & 69410 \\
\hline Fécondité forte & 19400 & 23809 & 29259 & 35811 & 43610 & 52807 & 63514 & 75774 \\
\hline Fécondité faible & 19137 & 23035 & 27696 & 33219 & 39587 & 46769 & 54709 & 63334 \\
\hline Fécondité constant & 19268 & 23528 & 28922 & 35736 & 44330 & 55158 & 68808 & 86020 \\
\hline
\end{tabular}

Source : Nations Unies : « Word population prospects », 2014

De nouvelles variétés du mil, sorgho et maïs résistant à la sécheresse sont adoptées, mais il semble improbable que la récolte suive le rythme de la croissance démographique. De manière récurrente, des millions de Nigériens sont confrontés à de graves pénuries alimentaires et le gouvernement du Niger demande régulièrement une assistance alimentaire d'urgence aux partenaires au développement et à la coopération bilatérale et multilatérale. Dans ce scénario, l'assistance alimentaire de la communauté internationale sera de plus en plus indispensable. Face au coût croissant des céréales et à la concurrence des économies émergentes (pour nourrir les animaux comme les humains), l'offre alimentaire extérieure risque toutefois de s'épuiser. Néanmoins dans le domaine de la sécurité alimentaire, l'initiative $3 \mathrm{~N}$ (les Nigériens Nourrissent les Nigériens) est le nouvel espoir de tout le peuple nigérien.Les réseaux sociaux assurés par les familles étendues et autres proches qui protègent actuellement les populations contre les chocs extérieurs s'effondreront vraisemblablement sous le poids du nombre grandissant de personnes glissant de la pauvreté chronique (nécessité d'emprunter de l'argent ou de dépendre de la famille étendue pour se nourrir pendant certaines parties de l'année) à la pauvreté persistante (dépendance annuelle continue de parents et organismes d'assistance extérieure, pour la nourriture et les premières nécessités).

Par ailleurs, il est difficile de prédire le réchauffement planétaire au niveau régional ou national (Kandji, 2006). Cependant, la plupart des scénarios climatiques peignent cependant un tableau sombre et même effrayant pour les pays du sahel et imagine que le Sahel va presque inévitablement droit à la catastrophe environnementale (N. U., 20l0).

Dans les 20 ans à venir, l'augmentation de la population ainsi que la nécessité d'améliorer la couverture sanitaire et les performances du système de santé, vont requérir des moyens considérables. 
Avec l'augmentation de l'espérance de vie et l'augmentation du nombre d'adultes et de personnes âgées, les cas de maladies cardio-vasculaires et de maladies liées au vieillissement vont augmenter.

Concernant le suivi des grossesses, leur nombre augmentera (un quasi doublement) d'ici 2030. Cela signifie que si l'on se fixe comme objectif que toutes les femmes en âge de procréer bénéficient de l'assistance de personnel qualifié lors de leur accouchement à l'horizon 2030, (contre une sur trois aujourd'hui), il faudra multiplier les moyens à mettre en œuvre au moins par six (6). Le nombre d'enfants de moins de 5 ans, qu'il faudra vacciner et dont il faudra suivre la croissance augmentera à peu près dans les mêmes proportions.

II est aussi fort peu probable que le Niger dispose de suffisamment de professionnels de la santé pour répondre aux besoins de sa croissance démographie rapide. Le pays est en retrait par rapport aux normes de l'OMS sur la taille de la population pour un médecin, une sage-femme, un infirmier. Comme le souligne le rapport 2005 de la Banque Mondiale sur le Niger: "Dans l'état actuel des choses, le système national de formation de ressources humaines pour le secteur de la santé n'est pas en mesure de répondre à de tels besoins ".

Au nombre de défis que laissent entrevoir cette croissance rapide de la population s'ajoute sans nul doute ceux liés à l'éducation, à la formation et à l'emploi du nombre d'enfants qui naitront. On peut, par exemple, se fixer comme objectif d'atteindre assez rapidement un taux net de scolarisation de $100 \%$ dans le primaire (près de $70 \%$ aujourd'hui) et d'ici 2030 un taux net de scolarisation de $60 \%$ dans le secondaire (moins de $15 \%$ aujourd'hui) et un taux brut de $15 \%$ dans le supérieur(moins de 1\% aujourd'hui), ce qui permettrait au Niger d'arriver à des niveaux d'éducation plus ou moins comparables à ceux observés aujourd'hui dans les pays émergents.

Ces objectifs correspondent à une augmentation de près $80 \%$ pour le primaire, à leur multiplication par près de 7 fois pour le secondaire et par 12 pour le supérieur. En outre, d'ici 2030, les populations scolarisables pour le primaire, le secondaire et le supérieur peuvent être multipliées par deux, au moins. La combinaison de ces facteurs conduit ainsi en 2030 à des effectifs d'élèves au moins trois fois plus nombreux au primaire, 15 fois plus nombreux au secondaire et 25 fois plus nombreux au supérieur. Atteindre ces objectifs constitue donc un défi qu'il ne sera pas facile de relever car les moyens humains et financiers qu'il faudra mobiliser sont très importants.

Par ailleurs, au rythme dont croît le nombre de demandeurs d'emploi, on peut aussi se demander si l'Etat sera en mesure de créer des emplois suffisants et mieux rémunérés.
Que peut- on dire au sujet de la structure de la population par sexe ? En examinant la répartition de la population par sexe on peut souligner qu'en 2012 , la proportion des femmes, par rapport à l'ensemble de la population dénombrée, est de $50,6 \%$ contre $49,4 \%$ pour les hommes. Les résultats de toutes les hypothèses font remarquer un retrait progressif très lent de la part des femmes. Toutefois, l'effectif de la population restera sensiblement partagé entre hommes et femmes (Tableau 3).

Nous pouvons souligner que la famille nigérienne et la dynamique culturelle représentent largement les intérêts masculins, beaucoup de femmes ne disposant d'aucune autonomie ou liberté de gestion de leurs maternités. Le pays n'a pas ratifié le Protocole de Maputo et sa proscription du mariage précoce. Pourtant, aucun développement ne serait envisageable avec la moitié de la population du pays. En conséquence, hier, aujourd'hui, demain, comme après demain, l'importance de l'égalité des sexes comme un moyen de donner aux femmes Nigériennes la possibilité de participer activement et de bénéficier du processus de développement le plus largement possible doit être reconnue dans la vie économique, sociale et politique. À cet égard, l'égalité des sexes consiste à s'assurer que le développement économique bénéficie des contributions réelles et potentielles que les femmes apportent à l'économie, et tirer le meilleur parti de la réserve de talents en veillant à ce que les hommes et les femmes ont une chance égale de contribuer au développement durable, améliorant ainsi leur bien-être et celui de la société.

Dans le domaine économique par exemple, étant donné le rôle clé des femmes dans le secteur agricole à travers le pays, améliorer leur accès à l'éducation, la formation, la technologie, la terre, le capital et les marchés, aboutirait à des progrès et des gains énormes pour cet important secteur de l'économie. Rendre l'agriculture et les investissements de développement rural dépendante des besoins et préoccupations des femmes doit donc faire partie intégrante de la transformation économique au Niger. C'est connu de tous, au Niger, les femmes sont majoritairement actives dans le secteur informel dynamique de l'économie nigérienne et spécialement dans des domaines tels que le petit commerce, la transformation alimentaire, le travail domestique et de ce fait l'amélioration des conditions de travail et l'accès aux services dans le secteur informel aiderait à réduire la pauvreté et l'insécurité chez les femmes et la société dans son ensemble. 
Tableau 3 : Evolution de la population par sexe selon l'hypothèse émise

\begin{tabular}{|c|c|c|c|c|c|c|c|c|}
\hline \multirow[b]{2}{*}{ Hypothèses } & \multicolumn{8}{|c|}{ Années } \\
\hline & 2015 & 2020 & 2025 & 2030 & 2035 & 2040 & 2045 & 2050 \\
\hline \multicolumn{9}{|l|}{ Homme } \\
\hline Fécondité médiane & 9718 & 11 834 & 14408 & 17478 & 21077 & 25233 & 29957 & 35239 \\
\hline Forte Fécondité & 9785 & 12032 & 14807 & 18141 & 22111 & 26793 & 32248 & 38497 \\
\hline Faible Fécondité & 9651 & 11637 & 14009 & 16816 & 20054 & 23704 & $2774 I$ & 32129 \\
\hline Fécondité constant & 9718 & 11889 & 14635 & 18103 & 22479 & 27997 & 34959 & 43743 \\
\hline \multicolumn{9}{|l|}{ Femme } \\
\hline Fécondité médiane & 9550 & 11588 & 14069 & 17035 & 20512 & 24524 & 29081 & $34|7|$ \\
\hline Forte Fécondité & 9615 & 11777 & 14452 & 17670 & 21499 & 26013 & 31266 & 37278 \\
\hline Faible Fécondité & 9486 & 11398 & 13687 & 16402 & 19534 & 23065 & 26967 & 31206 \\
\hline Fécondité constant & 9550 & 11639 & 14287 & 17633 & 21851 & 27161 & 33849 & 42276 \\
\hline \multicolumn{9}{|c|}{ Pourcentage de femmes dans la population totale } \\
\hline Fécondité médiane & 49,6 & 49,5 & 49,4 & 49,4 & 49,3 & 49,3 & 49,3 & 49,2 \\
\hline Forte Fécondité & 49,6 & 49,5 & 49,4 & 49,3 & 49,3 & 49,3 & 49,2 & 49,2 \\
\hline Faible Fécondité & 49,6 & 49,5 & 49,4 & 49,4 & 49,3 & 49,3 & 49,3 & 49,3 \\
\hline Fécondité constant & 49,6 & 49,5 & 49,4 & 49,3 & 49,3 & 49,2 & 49,2 & 49,1 \\
\hline
\end{tabular}

Source : Nations Unies : "Word population prospects », 2014

En ce qui concerne le domaine social, les gains de l'égalité des sexes peuvent être faits par des investissements dans l'amélioration et l'égalité des possibilités d'éducation pour les filles à tous les niveaux, l'accès des femmes aux ressources productives et aux opportunités d'emploi sûres, la fourniture d'infrastructures et services sociaux de base comme la santé maternelle, la santé des enfants et la protection sociale. Aussi, tout doit être fait à tous les niveaux de décision et de pouvoir politique pour protéger les femmes contre la violence domestique. Les hommes ont un rôle et une participation dans la promotion de nouveaux modèles égalitaire de la masculinité, dans lequel les femmes (et les hommes) sont capables de vivre à l'abri de la menace et le traumatisme de la violence domestique et sexuelle avec un meilleur accès à la santé, aux droits et à la dignité tout en l'améliorant simultanément la qualité de la vie des hommes.

La participation des femmes à la vie politique et publique à tous les niveaux de prise de décision au Niger doit être l'un des objectifs spécifiques autour duquel les buts et objectifs relatifs à l'égalité des sexes et l'autonomisation des femmes doivent être réglés. Au Niger, très souvent, les femmes n'ont pas une influence politique et, à ce titre, leurs besoins ne sont pas souvent pris en compte lors de la formulation des politiques et la conception et la mise en œuvre des programmes. Les femmes sont des agents de changement au sein de leurs familles, et augmenter leur voix et leur participation à la vie politique est essentiel pour faire progresser les problèmes des femmes dans les programmes nationaux, avec des avantages à la fois pour les femmes et les hommes.

Le bonus démographique : dividende ou bombe à retardement ? Le tableau 4 présente les ratios de dépendance selon l'hypothèse émise. Ces ratios sont obtenus par le rapport entre la population active (I564 ans) et la population inactive (moins de 15 ans et 65 ans ou plus). Sous l'hypothèse d'une fécondité constante on observera une augmentation du ratio de dépendance. II passera d'environ $1 \mid 2 \%$ à $118 \%$ entre 2015 et 2050. Par ailleurs, le ratio de dépendance est évalué entre $103 \%$ et $116 \%$ en 2030 selon l'hypothèse retenue. $\mathrm{Ce}$ fort taux de dépendance du Niger sape et sapera le potentiel d'accumulation des économies nécessaires à l'expansion de l'infrastructure du pays si des mesures appropriées ne sont pas prises.

On peut aussi faire remarquer, à l'exception de l'hypothèse de fécondité constante, une diminution constante du ratio de dépendance car les baisses supposées de la mortalité et de la fécondité entraineront une population active plus nombreuse mais où les jeunes adultes seront relativement plus nombreux. Une population active, surtout composée de jeunes, plus nombreuse est un bonus démographique qu'il convient de transformer en dividende démographique.

Si le pays parvient à planifier et à effectuer les investissements nécessaires dans les jeunes, il peut créer un cercle vertueux consistant à améliorer 
l'éducation, le capital humain et la productivité économique. Cela passe par l'éducation de la population en âge de travailler, son occupation d'emplois qui lui permet d'avoir une longue carrière productive avant qu'elle ne devienne à son tour âgée et dépendante. C'est la voie qu'ont choisi les Tigres Asiatiques, puis plus tard, de nombreux pays LatinoAméricains pour sortir de la pauvreté.

Comparé à d'autres pays de l'Afrique subsaharienne, la transition démographique du Niger est en retard, ce qui en effet réduit les perspectives de l'économie nigérienne à bénéficier des «dividendes démographique» issues de ce changement démographique supposée intervenir dans les générations à venir. Cependant, nous pouvons souligner que la croissance rapide de la population active impliquée dans la transition démographique du Niger que préconisent les trois hypothèses (forte, médiane et faible fécondité) posera de graves problèmes de développement mais aussi des opportunités.

Du point de vue du développement économique, une main-d'œuvre en pleine expansion impliquée dans la transition démographique du pays offre la possibilité d'accélérer la croissance économique et le développement social et, en outre, une population jeune peut être un atout formidable pour l'innovation et la créativité dans les économies et les sociétés.
Néanmoins, pour que le pays bénéficie de ce dividende démographique, les jeunes ont besoin d'être employée productivement et intégrés dans la société. Le pays a besoin d'une stratégie globale et des politiques judicieuses à offrir aux jeunes hommes et femmes avec des compétences et des connaissances pertinentes pour tirer profit de ces opportunités.

Cependant, dans le contexte actuel, le Niger est confronté à des défis croissants pour l'absorption significative des jeunes dans les systèmes éducatifs et les marchés du travail, et de ce fait ne bénéficie donc pas de dividende démographique issu de la jeunesse. Le Niger ne crée nulle part le nombre d'emplois nécessaires pour absorber les milliers de jeunes qui arrivent sur son marché du travail chaque année. II y a aussi la tendance difficile du chômage des jeunes diplômés, y compris les diplômés des établissements d'enseignement supérieur, qui se dessine dans le pays. Cela pose un sérieux problème en termes de politique de développement économique car elle contredit l'hypothèse selon laquelle l'enseignement supérieur et la formation augmentent la productivité et l'employabilité des jeunes. Ceci constitue aussi un gaspillage du produit du développement à cause du coût élevé de l'investissement dans l'enseignement supérieur et de la rentabilité sociale nulle des diplômés chômeurs.

Tableau 4 : Evolution du ratio de dépendance selon l'hypothèse émise

\begin{tabular}{|c|c|c|c|c|c|c|c|c|}
\hline \multirow[b]{2}{*}{ Hypothèses } & \multicolumn{8}{|c|}{ Années } \\
\hline & 2015 & 2020 & 2025 & 2030 & 2035 & 2040 & 2045 & 2050 \\
\hline Fécondité médiane & 111,6 & 111,5 & 111,0 & 109,0 & 104,8 & 99,7 & 94,1 & 88,4 \\
\hline Forte fécondité & 113,0 & 115,0 & 116,8 & 115,3 & 110,9 & 105,7 & 100,5 & 95,2 \\
\hline Faible fécondité & 110,1 & 108,0 & 105,2 & 102,7 & 98,6 & 93,6 & 87,7 & 81,6 \\
\hline Fécondité constante & 111,5 & $1 \mid 2,4$ & 114,3 & 116,4 & 117,3 & 117,6 & 117,7 & $7^{117,}$ \\
\hline
\end{tabular}

Source : Nations Unies : " Word population prospects ", 2014

Le manque de possibilités d'emploi face à la croissance de plus en plus rapide de la force de travail jeune peut être considéré, au Niger, comme une "bombe à retardement", tout comme le malaise des jeunes peut se traduire directement et indirectement dans des actions, y compris les conflits violents qui nuisent à la cohésion sociale et menacent la stabilité politique. La frustration et le ressentiment de n'avoir $\mathrm{pu}$ trouver un emploi comme prévu après les qualifications sont à l'origine de l'émigration des jeunes hautement qualifiés et par conséquent de la "fuite des cerveaux» du Niger. Du point de vue démographique à long terme, et à la lumière de la récente performance de croissance impressionnante, le Niger est confronté au défi de transformationd'une population jeune en un bonus de développement.
Renverser ces tendances appelle à un scénario de politique différent dans lequel la création d'emploi des jeunes en particulier est parmi les principaux objectifs du cadre macroéconomique et est une priorité pour la politique budgétaire. Ainsi, de nouveaux modèles de croissance sont nécessaires, $y$ compris des stratégies et des politiques industrielles qui favorisent la diversification économique et la création d'emplois de qualité. Le sens de l'urgence renouvelé pour prioriser l'emploi des jeunes dans les programmes de transition du Niger n'est pas seulement une question de répondre aux aspirations des jeunes pour une vie meilleure, mais aussi une nécessité pour améliorer le bien-être de la société nigérienne au sens large. 


\section{Discussions}

Après les contre-performances constatées dans la mise en œuvre des politiques de population et de santé de la reproduction, il est clair que l'option d'une "solution du marché " pour réduire la croissance démographique est peu envisageable au Niger du fait de l'inaccessibilité de la planification familiale à travers le pays. L'approche explicite utilisée " investissement limité " où les services de planification familiale sont assurés à travers les programmes de santé reproductive globaux dont l'objectif fondamental est l'amélioration de la santé, et non la réduction de la fécondité a fini par montrer ses limites, même si elle a permis d'éviter un rejet systématique de la planification familiale par certaines organisations religieuses.

La politique de recours à la planification familiale et la réduction de l'âge au premier mariage mais qui ne nie nullement l'importance de l'investissement dans le capital humain, l'amélioration de la santé, la réduction de la pauvreté et l'amélioration de la condition féminine s'adapte mieux au contexte nigérien. En effet, offrir une variété de méthodes contraceptives aux femmes désireuses de différer leurs maternités ou d'y mettre fin et contrer la désinformation qui circule au sujet de la contraception constituent un point de départ logique et nécessaire à la réduction de la fécondité. Bien que la demande de planification familiale déclarée au Niger soit faible, $37 \%$ seulement de cette demande est satisfaite (INS et ICF International, 2013). Les récents changements de politique adoptés au Niger, où les contraceptifs sont désormais gratuits et les voies de distribution plus larges, ont renforcé légèrement la prévalence contraceptive, mais le chemin à parcourir reste long.

Après plusieurs décennies d'inaction, toutefois, cette politique simplement axée sur un objectif d'accès généralisé à la planification familiale au Niger se trouve confrontée à de sérieux défis. L'absence d'infrastructure sanitaire et le manque crucial de personnels de santé à tous les niveaux limitent l'éventail d'options contraceptives pouvant être proposées. Aussi faut-il probablement s'attendre, pendant quelque temps encore, à voir plus de naissances évitées par les méthodes traditionnelles que par la contraception moderne.

L'accès à la planification familiale inclut la disponibilité d'une information exacte et culturellement appropriée. Beaucoup de femmes analphabètes et pauvres croient que la contraception est plus dangereuse que l'accouchement (Campbell et al. 2006). L'apport d'une information exacte aux femmes et aux couples n'est cependant pas sans difficultés non plus. Le recours à la radio et à la télévision pour la dissémination d'une information 2940 correcte sur la planification familiale et la rectification de la désinformation qui circule au sujet de la contraception doit être largement étendu. Soixantecinq pour cent des femmes de la capitale, Niamey, regardent la télévision; ce pourcentage est réduit à $2 \%$ dans les milieux ruraux (INS et ICF International, 2013).

Dans les endroits où la connaissance et l'accès à la contraception sont limités, les grossesses non planifiées seront courantes et une certaine proportion des femmes cherchera à les interrompre. Pourtant, l'avortement est illégal au Niger et aucune étude n'y a été menée sur la pratique de l'avortement non médicalisé. Notons qu'il existe des données limitées sur les communautés musulmanes du Nigéria, où une femme sur six déclare avoir eu une grossesse non désirée et près de la moitié d'entre elles disent avoir essayé d'interrompre cette grossesse, le plus souvent dans le but de mettre fin à leurs maternités ou de les espacer (Sedgh, 2006). L'Afrique occidentale, dans son ensemble, présente la plus haute mortalité imputable à l'avortement (I 40 pour 100 mille naissances vivantes par an) enregistrée dans le monde (Cleland et al. 20l I).

Par ailleurs, il est important de souligner que l'âge précoce au premier mariage est un facteur clé du nombre moyen d'enfants par femme du pays. L'âge médian au premier mariage est de 15,7 ans et l'âge médian à la première naissance est de 18,6 ans. Ces deux âges varient selon le niveau d'éducation. Ils sont respectivement 15,$6 ; 16,7$ et 21 , I puis 18,$3 ; 19,1$ et 22,7 ans pour les femmes sans instruction, de niveau primaire et de niveau secondaire ou plus (INS et ICF International, 2013). Ainsi, les filles qui se marient tôt ont aussi des enfants plus tôt que leurs pairs qui restent à l'école et se marient plus tard.

Augmenter l'âge de la maternité de cinq ans dans un pays tel que le Niger réduirait la croissance démographique future de 15 à $20 \%$ (Bruce et Bongaarts, 2009). Différer le mariage et la maternité allonge l'intervalle entre les générations et ralentit la croissance démographique. Les stratégies de rehaussement de l'âge au premier mariage sont limitées, mais il existe un consensus grandissant sur la nécessité d'un investissement accru dans les jeunes femmes. L'instruction des jeunes femmes renforce leur autonomie et leur capacité de gérer leurs maternités, profitant du reste à la génération suivante en ce que les mères instruites investissent dans leurs propres enfants. II est important d'adopter et de mettre en œuvre le plus rapidement possible des dispositions législatives et règlementaires interdisant le mariage précoce, en fixant par exemple l'âge au premier mariage des femmes à 18 ans. 


\section{Conclusion}

En examinant les enjeux sociaux, économiques et politiques associés à la croissance démographique du Niger, il est important de garder à l'esprit l'importance cruciale que revêt une stabilisation rapide de la population si l'on veut parvenir à un développement durable. Cette croissance démographique trop rapide peut être maîtrisée, de manière non contraignante, librement consentie par la population, si les autorités décident de s'engager résolument de manière claire dans cette voie.

Depuis l'adoption de la politique nationale de population en 1992, l'absence d'une application de mesures permettant de maitriser la croissance démographique, notamment l'accentuation de la planification familiale et l'amélioration substantielle de la scolarisation, a transformé un scénario démographique grave en une situation potentiellement catastrophique.

Le Niger doit assumer les erreurs du passé. L'examen objectif de la situation actuelle du Niger et de son évolution démographique prévisible fait de l'établissement d'un programme de planification familiale une stratégie politique indispensable. Si le Niger doit prendre des mesures significatives pour parvenir à la promotion de l'égalité des sexes et l'autonomisation des femmes, alors, comme le souligne l'OMS: «bien que la maîtrise de leur fécondité ne suffise pas en soi à gagner la pleine autonomisation et égalité sexuelle des femmes, il s'agit du premier pas et du plus important» (OMS, 2007).

La Déclaration du Gouvernement en Matière de Politique de Population de 2007 encourage un accroissement modeste des budgets de la planification familiale, mais il reste bien plus à faire. L'amélioration de la prévision des besoins contraceptifs et de la logistique est impérative, les ruptures de stocks de moyens modernes de contraception sont fréquents dans de nombreuses formations sanitaires du pays. La sécurisation des produits contraceptifs est plus qu'une nécessité.

II revient également de chercher à répertorier et d'éliminer tous les principaux obstacles qui s'opposent encore à l'utilisation des services de planification familiale. Le gouvernement du Niger, les organismes et les donateurs internationaux et tous ceux et celles voués à extraire les peuples de la pauvreté et à améliorer la santé des femmes et des enfants doivent impérativement s'unir en un effort vigoureux de sélection des meilleures pratiques des programmes de planification familiale d'antan et de mise en œuvre de ces pratiques au service du lancement d'une planification familiale volontaire à grande échelle.
Par ailleurs, il convient de mettre l'accent sur les aspects simples de la survie de l'enfant qui éviteront le décès des enfants de moins de cinq ans. Ces interventions de santé encourageront les familles à avoir moins d'enfants et ouvriront la voie vers la croissance économique.

Des efforts d'accroissement de l'âge au premier mariage doivent être entrepris, même s'ils ne produiront probablement pas de résultats substantiels dans l'avenir immédiat. Les lois contre les mariages précoces doivent être promulguées et appliquées. II est crucial d'investir dans des programmes et des politiques visant à mettre fin au mariage des enfants, à retarder la première grossesse, à espacer les naissances et à éviter une grossesse inopportune chez les adolescentes.

La nécessité d'un plus grand effort de scolarisation continue des filles, en particulier l'éducation secondaire, est urgente. La recherche montre que l'enseignement primaire aide les jeunes à lire et à écrire, mais que l'enseignement secondaire contribue à retarder l'âge au premier mariage et la grossesse et donne aux jeunes les compétences et la confiance nécessaires pour être efficaces sur le marché du travail. L'éducation favorise la baisse de la fécondité et est un investissement fondamental pour une économie plus forte. Les programmes d'éducation doivent préparer les étudiants au marché du travail du XXle siècle. Une plus grande participation au marché du travail permettra aux pays de récolter les fruits économiques du dividende démographique.

\section{Bibliographies}

Baidu-Foson J. et Napier T.L., 1998 -"Wind erosion within Niger", In Journal of Soil and Water Conservation, 53(2): pp. 120-125.

Bruce J. et Bongaarts J., 2009 -The new population challenge, In: A Pivotal Moment: Population, Justice and the Environmental Challenge (Mazur L, ed.), Washington DC: Island Press, pp. 260-275.

Campbell M., Sahin-Hodoglugil N.N. et Potts M., 2006 -Barriers to fertility regulation: a review of the literature, in Studies in Family Planning, 37(2) pp.87-98.

Cleland J., Ndugwa R. et Zulu E., 20II, - Family planning in Sub-Saharan Africa: progress or stagnation? In Bulletin of the World Health Organization, 89(2) pp. I37-I43.

Guengant J-P, 20II, Impact des politiques de population sur les politiques sectorielles et les évolutions démographiques au Burkina Faso, au Mali et au Niger. POPPOV Research Network 5ieme Conférence sur la recherche en Population, Santé de la reproduction et Développement économique 19-2I Janvier 20I I Marseille. 
Hodgson D et Watkins SC, 1997, Feminists and Neo-Malthusians: past and present alliances, Population and Development Review, 23(3):469523.

Institut National de la Statistique (INS) et ICF International, 2013 - Enquête Démographique et de Santé et à Indicateurs Multiples du Niger 20/2, Calverton, Maryland, USA : INS et ICF International, 320 pages.

Kandji S.T., Verchot L. et Mackensen J., 2006 Climate Change and Variability in the Sahel Region: Impacts and Adaptation Strategies in the Agricultural Sector,Nairobi, Kenya, World Agroforestry Center et UN Environment Programme, 56 pages.

OMS and All Party Parliamentary Group on Population, Development and Reproductive Health (APPG PD\&RH), 2007 -Return of the Population Growth Factor: It's Impact upon the Millennium Development Goals, London: APPG PD\&RH, 2007, 32 pages.

Potts M., Pebley A.M. et Speidel J., 2009, Editorial, Philosophical Transactions of the Royal Society in Biological Sciences, 364(I532): pp. 2975-2976.

Pritchett L.H., 1994 -Desired fertility and the impact of population policies, In Population and Development Review, 20(I) pp. I-56.

République du Niger, 1999 -Bilan Diagnostic des Actions et Perspectives dans le Domaine de la Population, de l'Environnement et de la Sécurité Alimentaire au Niger, Bureau de Réalisation
Technique d'Étude et de Conseil, Niamey, 67 pages.

République du Niger, Direction de la Population, Ministère du développement social, de la population et de la promotion de la femme, 1994 -projections démographiques 1994-2025, Niamey, 32 pages.

République du Niger, 2005 -projections de la population du Niger de 2005 à 2050, un appel à l'action, travaux démographiques, volume $\mathrm{n}^{\circ} \mathrm{I}, 98$ pages.

République du Niger, 2007 -Déclaration de Gouvernement en Matière de Politique de Population, Niamey, Niger, 8 pages.

Sedgh G., 2006 -Unwanted pregnancy and associated factors among Nigerian women, in International Family Planning Perspectives, 32(4), pp. I75-184.

Vallin J., 1995 -La démographie, collection Repères, Editions la Découverte, paris, 123 pages.

World Bank, Niger, 2005 - Providing All Nigeriens with Food, Education and Health Care, a Demographic Perspective, Washington, DC: World Bank, 160 pages.

Nations Unies- Division de la population, 2014 "Word population prospects", révision, 2012.

Nations Unies- Economic Commission for Africa, 2010 -Climate Change through the Lens of Vulnerability and Human Rights, presented at the Seventh African Development Forum (ADF VII) Pre-Event-, Addis Ababa, Ethiopia, 70 pages. 